developed the Surveillance Target Outpatient blood Pressure (STOP) decision support tool to prompt team members to communicate BP percentile to identify hypertensive patients. Objectives Improve recognition and intervention for elevated $\mathrm{BP}$ in ages $2-17$ in a paediatric nephrology clinic. 1.) $90 \%$ of visits utilise STOP form 2.) $90 \%$ of elevated BP percentiles have documented intervention

Methods Model for Improvement framework was employed for this paediatric resident initiated QI project including rapid cycle PDSA testing beginning January through August 2017. Multidisciplinary teammates included receptionists, CMAs, nurses, and physicians. Daily data collection included quantitative measures analysed using run charts and control charts.

Results STOP form usage increased from an average of 69\% to $92 \%(n=1498)$ using rapid cycle improvement by calculating and communicating BP percentile. Significant improvement was achieved in elevated BP management from an average of $74 \%$ to $100 \%$ of patients $(n=483)$ with decreased variability occurring over the span of the project.

Conclusions We successfully used QI methods and tools to develop and implement a team-focused decision support tool to standardise and improve identification and management of elevated BP percentiles. The STOP form can be adopted and utilised in numerous clinical settings to identify those at risk for hypertension

\section{REDUCED READMISSION RATES THROUGH MULTIDISCIPLINARY, WHOLE PERSON CARE}

Mary Zozaya-Monohon, Andrea Corona. Providence Health and Services, US

\subsection{6/bmjoq-2017-IHI.9}

Background Readmissions are a surrogate marker for a patient's ability to manage their health. Ambulatory care is poised to transform services by partnering with patients to enhance their success with disease management to reduce hospitalizations.

Objectives Empower patients to optimise whole person, selfcare with the support of a multidisciplinary team.

Methods A target population was selected after analysis of hospitalised and readmitted patients to confirm findings reported in the literature. A multidisciplinary team developed goals and objectives for whole person care to provide the framework for interventions. A process for identification, enrollment and discharge from the team was created. During

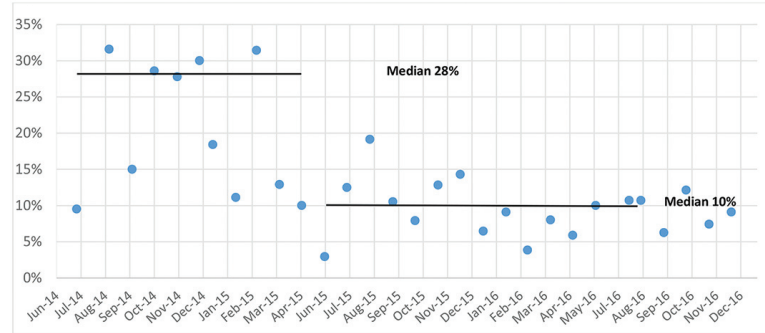

Abstract 902 Figure 1 Readmission rate run chart. The median readmission rate at baseline was $28 \%\left(20_{\text {th }}, 80_{\text {th }}\right.$ percentiles 11,31$)$ and decreased to $10 \%\left(20_{\text {th }}, 80_{\text {th }}\right.$ percentiles 6,13$)$. team meetings patient needs are prioritised and integrated services are coordinated. The electronic medical record supports communication to the primary care provider and other members of the healthcare team.

Results The median readmission rate at baseline was $28 \%$ and decreased to $10 \%$ post implementation. This reduction in readmissions was obtained by implementing the intervention in $20 \%$ of all admitted patients. Interventions were assessed at baseline in all discharged patients compared to post-implementation target population. Completion of Physician's Orders for Life-Sustaining Treatment (POLST) rose from 9\% to 41\%, Personal Health Questionnaire-9 and Generalised Anxiety Disorder screening significantly increased to 94\%. Evaluation by behavioural health and a pharmacist improved to $100 \%$.

Conclusions Readmission rates can be decreased through coordinated, whole person interventions on a targeted patient population in the ambulatory setting. Implementing multidisciplinary proactive planned care improves a patient's ability to care for themselves as demonstrated by a reduced readmission rate.

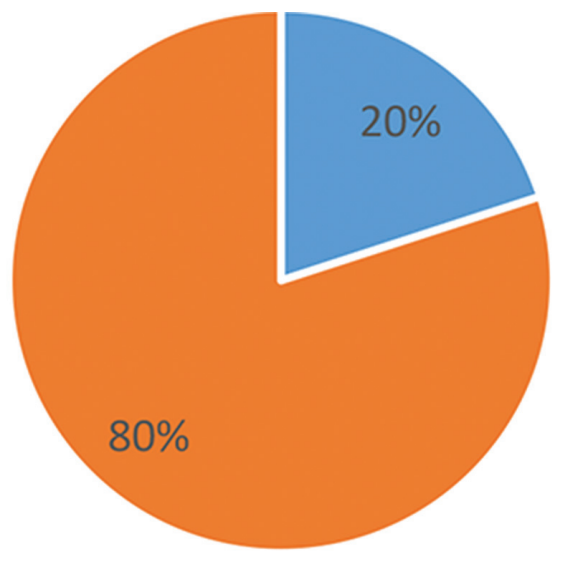

\section{TCM team All discharges}

Abstract 902 Figure 2 Percent of discharged patients receiving intervention

Abstract 902 Table 1 Interventions completed at baseline for all discharged patients compared to post-implementation in the target population

\begin{tabular}{lll}
\hline & $\begin{array}{l}\text { All discharged patients at } \\
\text { baseline }(\mathrm{n}=169)\end{array}$ & $\begin{array}{l}\text { Target-Population^ post- } \\
\text { intervention }(\mathrm{n}=94)\end{array}$ \\
\hline $\begin{array}{l}\text { Completion of } \\
\text { POLST }\end{array}$ & $15(9 \%)$ & $38(41 \%)$ \\
PHQ-9 Screening & $14(8 \%)$ & $88(94 \%)$ \\
GAD-7 Screening & $0(0 \%)$ & $88(94 \%)$ \\
Behavioural Health & $15(9 \%)$ & $94(100 \%)$ \\
Evaluation & & $94(100 \%)$ \\
Pharmacist & $7(4 \%)$ & \\
Evaluation & & \\
\hline
\end{tabular}

$\wedge$ Readmission within 90 days or a hospitalisation with a comorbid diagnosis of chronic obstructive pulmonary disease, diabetes, or heart failure. 